


\section{Sémiotique de la politique et systèmes non verbaux: la relation médias- politiciens grecs au temps de la crise economique}

$$
\text { 謩 }
$$

BY: Evangelos Kourdis
ARTICLE INFO:

\begin{tabular}{l}
\hline Volume: 06 \\
\hline Issue: 02:2020 \\
\hline Month: March \\
\hline ISSN: 2459-2943 \\
\hline DOI: 10.18680/hss.2020.0024 \\
\hline Pages: 117-139 \\
\hline Lic.: CC BY-NC-ND 4.0 \\
\hline
\end{tabular}

KEYWORDS:

political semiotics

Greek crisis

code of gesture

dress code

proxemics code

\section{ABSTRACT}

uring the Greek economic crisis, the European media engaged in a series of press articles, television reports, and press cartoons particularly critical of the behavior of the contemporary Greek and the quality of the politicians who govern him. This attitude intensified during the radical left government of the SYRIZA party from January to August 2015. In this period, the European media engaged in a semiotic devaluation, an unprecedented deconstruction of Greek politicians' social image of the SYRIZA party's radical left government. The European Union authorities recorded this deconstruction at the level of the three semiotic codes: that of gesture, that of the dress code, and that of proxemics. This study proposes to analyze in a semiotic approach these choices expressed through polysemiotic texts likely to interpret a conscious manipulation of European public opinion, also introducing uses previously unknown in the political and media culture of Europe.

\section{Introduction}

Au cours de la crise économique grecque (2010-2018), les médias européens se sont engagés dans une série d'articles de presse, de reportages télévisés et de caricatures de presse particulièrement critiques à propos du comportement du Grec contemporain et de la qualité des politiciens qui le gouvernent. Indépendamment de la véracité ou non de ces publications, il semblerait que le monde européen de la communication ait perdu de la qualité qui le distinguait jusqu'alors en se livrant en 2015 à une dévaluation, une déconstruction sans précédent 
de l'image sociale des politiciens grecs du gouvernement de gauche radicale du parti SYRIZA. Pour la majorité des citoyens grecs, les choix politiques retenus ridiculisaient les décisions politiques pris par leur gouvernement et toutes les relations avec les créanciers de la Grèce et les autres Etats membres de la zone euro. Alonso (2014) constate que:

on retrouverait dans le discours économique et politique un récit pour rendre compte de la crise de l'économie grecque construit de la manière suivante: des événements (la découverte du déficit abyssal de l'économie grecque, par exemple, avec ses effets d'intensification dramatique et passionnelle) jalonnent et rythment un cycle et l'orientent dans un sens ou dans un autre (alors on raconte l'inexorable décadence et la supercherie de l'économie hellénique depuis quelques années) avant de l'intégrer dans une temporalité plus étendue, une structure culturelle et sociale (et alors on affirme la fragilité endémique des institutions ou la corruption structurelle des pays du sud). (Alonso 2014: 6465)

Dans cette situation, les médias européens ont joué un rôle qui est considéré par une partie de l'opinion publique grecque comme 'ambigu' si on tient compte de la façon dont les médias ont présenté une série d'événements qui concernaient la vie politique grecque. Pour une partie de l'opinion publique, il y avait une dimension sémiotique dans la présentation de ces évènements politiques qui connotait un mépris des partis politiques européens dits 'traditionnels' (Droite et Centre) vers les décisions politiques et les déclarations que le gouvernement de gauche radicale du parti SYRIZA souhaitait suivre. Cette dimension sémiotique de la communication médiatique était déjà soulignée par Lamizet qui disait que "le rôle des médias est donc primordial dans la structuration de l'événement: c'est dans les médias qu'il acquiert, pour la première fois, la consistance d'un signe. En mettant en œuvre une représentation de l'événement, les médias lui donnent une dimension sémiotique" (Lamizet 2006: 21).

Cette étude se propose d'analyser dans une approche sémiotique ces choix exprimés à travers des textes polysémiotiques susceptibles de traduire une manipulation consciente de l'opinion publique européenne, introduisant ainsi des usages inconnus jusqu'alors dans la culture politique et médiatique de l’Europe.

\section{La sémiotique de la politique}

Il est généralement connu que la politique suit des règles de communication précises surtout devant la caméra journalistique. Les politiciens, hommes et femmes, prêtent attention à la façon dont ils / elles s'habillent, s'asseyent, se comportent. Ils / elles doivent respecter certaines normes dans le cadre des déontologies professionnelles, des codes 
et des rites du savoir-vivre politique. Comme Barthes (1957: 150) le constate, l'image d'un politicien "propose un climat physique, un ensemble de choix quotidiens exprimés dans une morphologie, un habillement, une pose." Pour certains citoyens, les politiciens sont une sorte de symbole ou doivent être une sorte de modèle parce qu'ils font partie de la vie publique qui contraint à des normes précises.

C'est la violation de ces normes précises qui provoque une suite de reportages journalistiques qui visent aux politiciens grecs du gouvernement de gauche radicale $\mathrm{du}$ parti SYRIZA. Il ne s'agit pas d'un événement inattendu. Pour Barthes (1980: 150), “le Politique est, subjectivement, une source continue d'ennui et/ou de jouissance; c'est, de plus et en fait (c'est-à-dire en dépit des arrogances du sujet politique), un espace obstinément polysémique, le lieu privilégié d'une interprétation perpétuelle (si elle est suffisamment systématique, une interprétation n'y sera jamais démentie, à l'infini)." Poutant, la question qui se pose est de savoir les limites de ces interprétations perpétuelles sinon nous traiterons un cas de sémiosis illimitée.

Lamizet (2002: 267) avance plus en soutenant que "[1]'indentité des acteurs politiques se fonde moins sur l'image qu'ils donnent ou sur le discours qu'ils énoncent ${ }^{1}$ que sur les formes symboliques et les représentations initiées par leurs pratiques institutionnelles et par leurs choix stratégiques." Ces formes symboliques et les représentations initiées par leurs pratiques institutionnelles sont souvent manipulées par les médias qui influencent pour leur part la sémiotique de la politique.

Bronwen \& Ringham (2006: 150) définissent la sémiotique de la politique comme une branche de la sociosémiotique qui s'intéresse à l'analyse du discours politique, des institutions et des stratégies de persuasion ainsi que du rôle des acteurs individuels. Elle comprend l'étude de phénomènes politiques tels que la construction de l'opinion publique et l'organisation et le fonctionnement de campagnes électorales. Les chercheurs se sont particulièrement intéressés aux représentations du pouvoir dans les médias et à la dimension politique du discours institutionnel ${ }^{2}$.

Dans la définition de la sémiotique ci-dessus ce qui manque c'est la notion de signe. Il est à noter que la politique publique est un espace de signes et c'est pour cela que la sémiotique politique tant pour Turpin que pour Lamizet est définie en tant qu'élaboration des signes.

\footnotetext{
${ }^{1}$ Pour Bordron (2013), "la tradition des photographies politiques montre que l'image peut cacher (ne pas dire) et même effacer, ajouter abusivement, donner pour réel un montage de studio, etc. Le point essentiel, dans tous ces cas, n'est pas la vérité de l'énoncé mais bien le mode véridictoire de son énonciation. Il semble bien qu'il y ait une convention du 'montrer vrai', de la rectitude iconique, équivalent au 'dire vrai' du langage."

2 Toutes les traductions de l'anglais et du grec sont nos traductions.
} 
Plus précisément, pour Turpin:

la sémiotique politique est l'étude des signes du politique et de la manière dont ils produisent du sens. Le champ ainsi défini est très large si l'on considère l'importance des manifestations signifiantes du politique: rites, discours, mais aussi institutions qui peuvent relever de cette étude [...]. (Turpin 2006: 286)

Lamizet (2006b) révèle encore une dimension, celle des pratiques sociales. Quant à Lamizet (2006b: 4), “[...] la sémiotique politique élabore une logique de signes fondés sur l'articulation entre des signes, de toutes sortes, et des pratiques sociales mises en œuvre par des acteurs sociaux dans l'espace publique." Ces définitions reflètent le cadre d'étude des événements politiques, des textes politiques, que nous allons étudier. Nous rappelons ici que pour l'Ecole sémiotique de Moscou-Tartu, "la recherche sémiotique contemporaine traite le texte comme une des notions fondamentales en le considérant non pas comme un objet stable ayant des traits constants mais comme une fonction. Tout peut être considéré comme texte: l'œuvre toute entière, sa partie, le groupe de composition, le genre - bref, toute une littérature" (Lotman 2004 : 152-153).

\section{Les systèmes sémiotiques non verbaux}

La sémiotique de la politique a pu se développer profitant de l'expansion de la notion de texte et de discours à la politique. Comme Ahonen (1991: 264) le soutient, "l'analyse de la génération de sens en politique reste [...] déficiente si la recherche néglige la substance non verbale en tant que porteur de la politique [...]." Il est vrai que les dernières décennies l'étude sémiotique de la politique semble se concentrer moins aux messages linguistiques en comparaison avec le passé. La communication politique non verbale paraît être plus révélatrice au niveau de sens que les déclarations langagières et la sémiotique peut jouer un rôle important dans ce sens. Comme Drechsler le soutient :

la Science Politique telle qu'elle existe, spécialement ayant une fixation glottocentrique [...] a besoin d'un système systématique, scholastique, théoriquement riche et englobant tous les aspects non glottocentriques de chaque champ. La Sémiotique da la politique peut accomplir cela, à condition que la sémiotique ne soit pas trop exclusivement glottocentrique elle-même. (Drechsler 2009: 91) 
En plus, Lamizet et Silem (1997: 506) mentionnent qu'“'avec le développement des moyens de communication non linguistiques, la sémiotique, en tant que réflexion sur les systèmes de signes ou de significations, s'est affranchie de la réflexion linguistique et a créé ses propres outils." ${ }^{3}$ Nous allons voir que la différentiation du comportement politique des autorités de l'Union Européenne se fait au niveau de la communication non verbale et plus précisément au niveau de trois systèmes sémiotiques non verbaux qui se lient entre eux: celui de la kinésique (système gestuel/haptique), celui de code vestimentaire (système de vêtements) et celui de la proxémique (système de distance).

\subsection{Le code gestuel}

En ce qui concerne le premier système sémiotique, celui de la gestualité, Greimas \& Courtés (1993: 165) le relie “[...] au phénomène paralinguistique qui aurait une fonction d'auxiliation dans le cadre de la communication intersubjective." Plus précisément, comme Danesi le définit:

le langage du corps est le terme général utilisé pour indiquer une communication au moyen de gestes, des positions du corps et d'autres signaux et signes corporels volontaires et involontaires. Cela inclut également les habitudes de toilettage, les styles de coiffure et de vêtements, les pratiques, comme le tatouage et le perçage corporel. (Danesi 2004: 53) ${ }^{4}$

L'importance de la gestualité dans la communication politique est indéniable. Calbris (2001: 130) souligne que “[c]e n'est pas un hasard si le geste précède la verbalisation. Il exprime la pensée, la plupart du temps, avant la parole elle-même. Non seulement il la prépare, mais il la complète d'avance." Selon Danesi (2004: 53), "le langage corporel communique des informations non-dites sur l'identité, les relations et les pensées des personnes, ainsi que leurs humeurs, motivation et attitudes." 5 Basso Fossali (2017:4) révèle le caractère sémiotique du geste en mentionnant qu'“à travers le geste, on sculpte localement une niche de sens qui s'interpose entre la rétroduction énonciative (genèse de l'acte) et la prospection des conséquences (efficacité de l'acte)."

Le code tactile (ou haptique), la communication par le toucher, est une composante essentielle dans la gestualité. D'après Danesi:

\footnotetext{
${ }^{3}$ Alonso fait une autre constatation. D'après Alonso (2014: 62), “[...] la sémiotique semble parfois laisser à d'autres disciplines le soin d'utiliser ou de manipuler certains de ses concepts. Pour ces disciplines - comme le marketing, politique ou commercial -, la sémiotique n'est qu'une sorte de boîte à outils dans les meilleurs des cas ou tout simplement le fournisseur de quelques concepts de schématisation simplificateurs ou de notions passe-partout qui se répandent avec une plus ou moins grande pertinence et efficacité [...]".

4 "Body language is the general term used to indicate communication by means of gestures, postures, and other witting and unwitting body signals and signs. It also includes grooming habits, hair and clothing styles, and such practices as tattooing and body piercing"' (Danesi 2004: 53).

5 "Body language communicates unspoken information about people's identity, relationships, and thoughts, as well as moods, motivation, and attitudes" (Danesi 2004: 53).
} 
dans la plupart des cultures, une forme de salutation de base implique une poignée de main, qui est un exemple parfait d'un comportement social régi par un code tactile, c'est-à-dire par un code qui régit les modèles de contact dans les situations interpersonnelles. L'étude du toucher passe sous la rubrique de 1'haptique. (Danesi 2004: 55) ${ }^{6}$

Il est à noter que selon Heslin (1974), la communication haptique, à l'exclusion des comportements ouvertement hostiles tels que les gifles ou la fessée, peuvent être classés en cinq types principaux : fonctionnel/professionnel, social/poli, amitié/cordialité, amour /affection, sexuel/passion.

Nous allons constater qu'à l'exception des deux dernières catégories, il y a une grande difficulté à catégoriser le comportement des officiers de l'Union Européenne vers les politiciens grecs en question. Nous allons aussi voir par la suite que la gestualité entre les fonctionnaires de l'Union Européenne et les membres du gouvernement grec de l'époque dépassait de beaucoup les limites du savoir du comportement politique.

\section{2. Le code vestimentaire}

Pour la sémiotique, le vêtement et ses accessoires, la mode, sont porteurs de sens. Selon Barthes (1967 : 45), "le vêtement est l'un de ces objets de communication, comme la nourriture, les gestes, les comportements, la conversation [...]." Lotman (2004: 172) révèle son caractère culturel affirmant que "telle est, en fait, la fonction de la mode: elle est destinée à être le métronome et le catalyseur du développement culturel." Nous allons voir par la suite que le code vestimentaire des membres du gouvernement grec de l'époque a donné naissance à des commérages qui ridiculisaient le gouvernement grec et le premier ministre même. Selon Danesi:

les vêtements sont plus qu'une simple couverture corporelle destinée à protéger. C'est un système de signes qui est interconnecté avec les autres systèmes de signes de la société à travers lesquels nous pouvons envoyer des messages sur nos attitudes, notre statut social, nos convictions politiques, etc. (Danesi 2004: 178) ${ }^{7}$

Ainsi, Danesi (2004: 177) donne l'exemple d'un homme qui s'intéresse à travailler dans une banque et qui doit passer un interview. Danesi remarque que sa cravate doit être d'une couleur conservatrice et que son utilisation peut être optionnelle.

\footnotetext{
6 "In most cultures, a basic form of greeting involves handshaking, which is a perfect example of a social behavior regulated by a tactile code, that is, by a code that governs the patterns of touch in interpersonal situations. The study of touch goes under the rubric of haptics" (Danesi 2004: 55).

7 "Clothing is more than mere bodily covering for protection. It is a sign system that is interconnected with the other sign systems of society through which we can send out messages-about our attitudes, our social status, our political beliefs, etc." (Danesi 2004: 178).
} 
Dans la communication politique la cravate semble être un accessoire imposé pour les situations dites sérieuses ou formelles. ${ }^{8}$ Assez souvent, les politiciens, pour donner l'impression d'une ambiance cordiale, ne portent pas de cravate lorsqu'ils passent à des situations de communication politique non formelles ce qui semble être un acte accepté et justifié après des discussions longues provoquant l'ennui. En particulier la cravate est liée au conservatisme. Le journaliste Florian Delafoi (2017) considère comme "une petite révolution" le fait que le parlement britannique ait assoupli son code vestimentaire, acceptant que la cravate ne soit plus obligatoire.

\section{3. Le code proxémique}

La proxémique est un système sémiotique pas largement étudié. Greimas \& Courtés (1993: 300) définissent la proxémique comme un champ de recherche "[...] qui vise à analyser les dispositions des sujets et des objets dans l'espace, et, plus particulièrement, l'usage que les sujets font de l'espace aux fins de signification." Selon Fabbri (1968: 7374), «on devrait également étendre le geste proxémique jusqu'à la définition d'un domaine des distances de relations sociales limitée à deux partenaires (dyades). Il est possible que ces relations soient articulées sur la catégorie spatiale universelle symétrie vs dissymétrie». Fabbri nous explique que:

La symétrie serait reliée à la proximité = près (à la sympathie, aux interactions fréquentes et intimes, à l'usage mutuel du tu. La dissymétrie serait reliée à la distance $=$ loin (l'indifférence et l'antipathie, les interactions rares et formelles, l'usage mutuel de vous); ou aux relations spatiales avant vs après ou dessus vs dessous en se rattachant alors à l'idée de supériorité et d'infériorité (à l'influence et le pouvoir vs soumission, et à l'emploi dissymétrique du tu et du vous). Il en résulterait un modèle généralisé des relations qui rattacherait les universaux des statuts et des affinités à la dimension spatiale interpersonnelle. (Fabbri 1968: 74)

Nous allons voir que la manipulation de ces systèmes sémiotiques lors les rencontres politiques entre des hommes politiques grecs et les autorités de l'Union Européenne a contribué à la transmission par les médias européens d'une certaine confusion en ce qui concerne les intentions bienveillantes de ces derniers pour aider la Grèce à sortir de la crise économique de l'époque. Une confusion qui était difficile d'être interprétée même par les agents de la communication médiatique, les journalistes.

Si on tient compte que, selon Lamizet (2006b: 13), "la communication politique est toujours une communication destinée" (emphase dans l'original), l'inquiétude des agents

\footnotetext{
${ }^{8}$ Mayi (2016) constate que le costume avec cravate reste jusqu'aujourd'hui un choix vestimentaire commun pour les hommes politiques français. Parmi les connotations du code vestimentaire dans la vie politique il y a le sérieux et la diligence des hommes politiques.
} 
de la communication politique était justifiée à cause du caractère imprédicable du comportement politique des agents de l'Union Européenne. Comme Lotman (2013: 67) le mentionne, "l'élément imprédicable devient un acte, il est indéniablement soumis à interprétation et on l'attribue d'une motivation additionnelle après l'événement." Et cela se fait parce que:

les sujets de l'énonciation s'y expriment au nom de, ils n'expriment pas une parole ou une position singulière, mais leur activité symbolique est toujours située - pour eux-mêmes et pour les autres - par rapport à une institution ou à une dimension institutionnelle dont ils se légitiment. (emphase dans l'original) (Lamizet, 2006b: 13)

C'est cette symbolisation des actes politiques qui sera le but de cette étude tenant en compte que le comportement des dignitaires européens envers les officiels grecs est devenu objet d'analyse pour les grecs. En plus, ce comportement ait jailli le soupçon d'un complot contre la Grèce organisé par les créanciers du pays (surtout par l'Allemagne) pour affaiblir et ridiculiser le peuple, combattre sa résistance contre les mesures économiques exhaustives imposées. ${ }^{9}$

\section{Quelques exemples des événements politiques sémiotiques du passé}

La question qui en résulte est pourquoi doit-on chercher des aspects sémiotiques aux systèmes sémiotiques non verbaux du moment où une interprétation de la communication non verbale peut être accidentelle? La réponse pourrait être la fréquence de ces évènements non verbaux, une fréquence qui connote une certaine intentionnalité. Pourtant, l'exploitation des moyens non verbaux dans la vie politique européenne était assez limitée étant donné que le haut niveau de la culture européenne ne permettait pas la projection publique des événements de ce type par les médias.

Ainsi, nous savons que, le Président de la République Française Nicolas Sarkozy, pour exprimer son désaccord avec l'intention du Premier Ministre grec Georges Papandréou d' organiser en 2011 un référendum contre les mesures d'austérité proposées par les dirigeants de la zône euro, ne l'avait pas accueilli à l'entrée du bâtiment où un sommet se réalisait à Cannes. Mais, en plus, il n'avait pas quelqu'un pour l'orienter dans le bâtiment ce qui avait été interpeté par les médias comme un acte impoli intentionnellement enregistré pour exprimer le mécontentement du Président français. Pourtant, l'évocation du hasard peut justifier cet événement qui s'était déroulé loin des caméras journalistiques.

\footnotetext{
${ }^{9}$ Voir Kourdis (2016).
} 
Cependant, quand un incident politique se fait devant les caméras journalistiques cela change. Ainsi, l'ambassadeur turc avait été humilié en 2010, devant les photographes et les cameramen, par le Ministre israélien de la Défense Danny Ayalon, en signe de protestation contre la diffusion sur une chaîne de télévision privée turque d'une série jugée antisémite par Israël. Ce dernier avait notamment refusé de lui serrer la main, le contraignant à attendre longuement dans un couloir, puis à s'asseoir plus bas que ses interlocuteurs. ${ }^{10}$

Dans ce cas, les codes gestuels et proxémiques, tout comme aussi l'aménagement dans l'espace, ont crée des connotations négatives qui ont provoquées des déclarations dénotatives pour affronter le problème crée. Mais qu'en est-il quand des événements pareils basés sur les codes non verbaux se répètent, se multiplient devant les caméras journalistiques donnant ainsi l'occasion de produire des interprétations ambiguës, même négatives, pour la bienveillance des dignitaires européens-créanciers.

\section{La dévaluation d'une idée}

Selon Danesi (2004: 53), les signes kinésiques peuvent être innés (involontaires), acquis (volontaires) ou un mélange des deux. La dimension sémiotique dans l'étude de la communication politique concerne principalement la deuxième catégorie car il semblerait que les signes qui caractérisaient le comportement politique des autorités de l'Union Européenne vers les politiciens grecs étaient volontaires. Ainsi, pendant la première période du gouvernement de la gauche radicale SYRIZA qui a duré neuf mois (Janvier-Août 2015) le public européen s'est trouvé devant une dévaluation sémiotique de ce gouvernement, à notre avis sans précédent dans l'histoire politique européenne. Cette situation n'est pas passée inapercue par la presse grecque. Ainsi, le journal en ligne grec iefimerida publie le 5 Février 2015 un article ayant comme titre "Pourquoi les dirigeants européens oublient-ils leurs bonnes manières quand ils se retrouvent à côté de membres du gouvernement grec ?"11 Cet article critique contient des photos et des commentaires $^{12}$ sur le comportement des dirigeants de 1'Union Européenne.

\footnotetext{
${ }^{10}$ Voir, https: / / bit.ly / 2Ntdgzu (dernière consultation le 26.11.2020).

${ }^{11}$ A voir, https:/ / bit.ly / 3u204SW (dernière consultation le 16.12.2020).

${ }^{12}$ Pour Barthes (1961 : 137), “aucune photographie n'a jamais convaincu ou démenti personne (mais elle peut 'confirmer'), dans la mesure où la conscience politique est peut-être inexistante en dehors du logos : la politique, c'est ce qui permet tous les langages."
} 
Les messages iconiques selectionnés proviennent des revues grecques et étrangères, imprimées et électroniques qui ont circulé pendant la première période du gouvernement de la gauche radicale SYRIZA en Grèce qui a duré neuf mois (Janvier-Août 2015).

Plus précisément, la première photo concerne l'arrivée du politicien Allemand Martin Schultz, président du Parlement européen, à Athènes le jeudi 29 Janvier 2015. Ce jour-là, il pleuvait, mais le Premier Ministre Alexis Tsipras a descendu les marches du perron de sa résidence officielle de Maxime pour aller à sa rencontre. Entre sourires et poignées de main, la main de Sultz se pose sur le col de Tsipras (fig.1). Le journaliste commente qu'avec ce geste, d'une intimité incroyable envers un Premier Ministre, M. Sultz avec humour soulignait l'absence de cravate, ${ }^{13}$ un acte de rébellion politique pour Tsipras.

Il est à noter que le Premier Ministre grec avait déclaré le 24 janvier 2015 qu'il ne porterait une cravate que lorsque la Grèce quitterait les programmes d'aide économique qu'elle recevait des pays membres de la zône euro. ${ }^{14}$ En plus, dans une interview au journal américain The New York Times le 17 janvier 2015, Tsipras reliait sa décision de ne pas porter de cravate à sa idéologie politique en mentionnant: “je pense que s'il y a quelque chose que les gens apprécient dans Syriza et moi-même, c'est que nous n'avons pas assumé la mentalité des partis établis et leurs façons spécifiques de s'habiller, d'agir."15

Un autre journaliste du même quotidien remarque que ce choix vestimentaire est aussi adopté par d'autre membres du gouvernement grec. Ainsi, Yanis Varoufakis, le nouveau Ministre grec des Finances, a rencontré le lundi 2 février 2015 le Ministre britannique des Finances George Osborne, en chemise bleue, veste en cuir et sans cravate (fig.2). Panos Kammenos, le nouveau ministre de la Défense et ancien porteur de cravate, a également commencé à abandonner l'accessoire. ${ }^{16}$ Sullivan (2019) supporte que:

dans la gauche européenne contemporaine, le radicalisme anti-austérité combiné à la perturbation vestimentaire est devenu une dominante culturelle. Les attitudes non conventionnelles à l'égard de l'habillement, qui se manifestent par des choix de style, sont devenues emblématiques d'un rejet du statu quo et des défis politiques posés par des insurgés et des opposants aux poli-

\footnotetext{
13 Pour Barthes (2001: 180), “toujours pour les hommes ou pour les adolescents, pendant très longtemps, le cou a été noué, fermé par une cravate, la cravate ayant pour fonction, précisément, de cacher le cou," mais pour Owyong (2009: 204), la cravate se distingue modalement et elle est fréquemment le point d'entrée visuel dans le costume connotant un pouvoir, une autorité, un décorum inébranlable.

${ }^{14}$ Boutin, Dard et Rouvillois (2019) relie cette attitude de Tsipras au populisme et ils mentionnent que "le populiste se manifeste enfin jusque dans la transgression des rites de l'ancien monde: c'est ainsi qu'après sa victoire de janvier 2015, Tsipras avait refusé de prêter serment sur la Bible, conformément aux usages traditionnels, et qu'il affiche comme un acte de rébellion politique son refus obstiné de porter une cravate."

${ }^{15}$ A voir, Daley (2015).

${ }^{16}$ A voir, Friedman (2015).
} 
tiques établies jusqu'alors, de Pablo Iglesias, le chef du parti populiste de gauche Podemos en Espagne à Alexis Tsipras et Yanis Varoufakis du parti réformiste de la gauche radicale grecque, Syriza. (Sullivan, 2019: 167)17

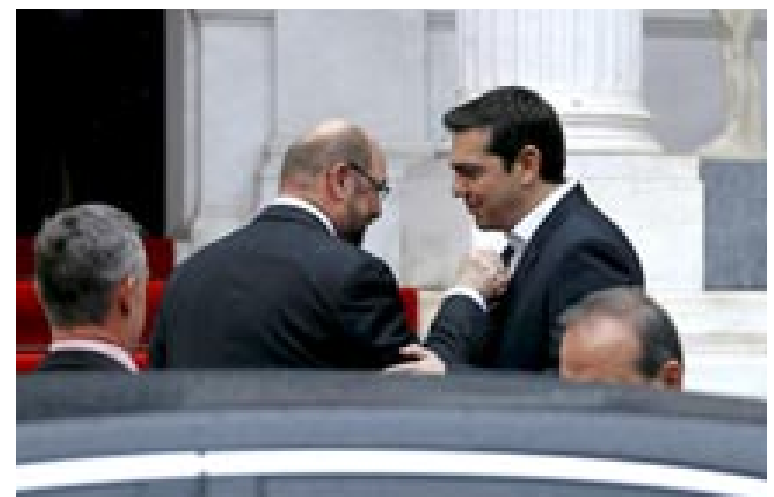

Figure 1. Visite du Président du Parlement Européen Martin Schulz à Athènes (29.1.2015)

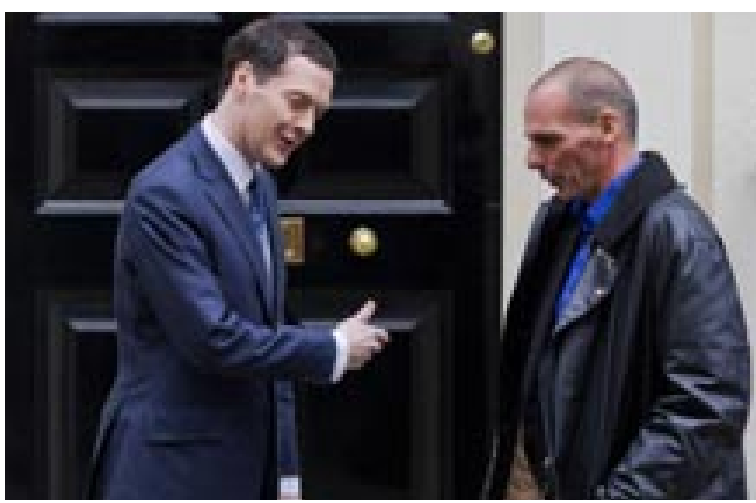

Figure 2. Yanis Varoufakis et George Osborne à Londres (2.2.2015)

Un jour avant sa visite à Londres (le dimanche 1.2.2015), le Ministre grec des Finances, Yanis Varoufakis, accompagné du Vice-ministre grec des Relations Economiques Internationales, Euclide Tsakalotos, rendirent visite au Ministre français des Finances Michel Sapin à Paris. Le Ministre français prit un bout du foulard de M. Tsakalotos et le lui enroula autour du cou en lui demandant pourquoi il le portait (fig.3). Le journaliste étonné commente l'attitude du ministre jouant avec le foulard d'un ministre d'un autre pays devant les caméras, lors d'une réunion officielle. Le journaliste ne dit pas que peut-être c'était un geste qui connote l'anticonformisme des ministres grecs de la gauche radicale pour lesquels la cravate est un symbole des partis politiques conservateurs et que c'est à cette idéologie à laquelle renvoie le ministre français faisant remarquer l'absence de la cravate qui fait partie du protocole politique et demandant au Ministre grec s'il fait froid à Paris.

Le Vice-ministre grec Euclide Tsakalotos n'était pas le seul face au comportement du Ministre français des Finances, Michel Sapin. Ce dernier arrivant à une conférence de presse commune tient par le bras le Ministre grec des Finances Yanis Varoufakis ce qui connote une grande familiarité (fig.4). Une atmosphère de joie ou une convivialité entre deux hommes, beaucoup plus qu'entre deux politiciens, rien ne justifiant une telle image devant les médias. Il semble que le sourire est le seul 'dispositif' pour dépasser un moment embarrassant.

\footnotetext{
17 "On the contemporary European left, anti-austerity radicalism combined with vestimentary disruptiveness has emerged as a cultural dominant. Unconventional attitudes to dress, manifested as style choices, have become emblematic of a rejection of the status quo and the political challenges mounted by insurgent, anti-establishment figures, from Pablo Iglesias, the leader of the populist left Podemos party in Spain, to Alexis Tsipras and Yannis Varoufakis of the Greek radical left reformist party Syriza" (Sullivan, 2019: 167).
} 


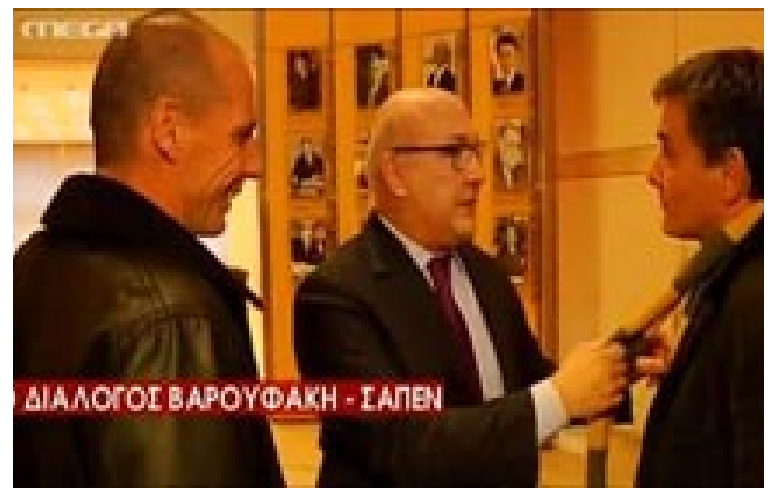

Figure 3. Las visite de deux Ministres grecs à Paris (1.2.2015)

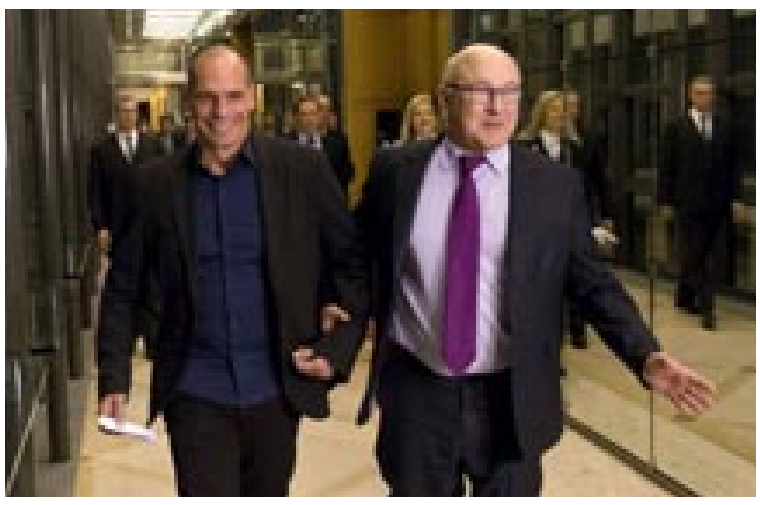

Figure 4. Michel Sapin et Yanis Varoufakis $(1.2 .2015)$

Le journaliste de iefimerida appelle ces gestes des 'plaisanteries' et il commente que ces plaisanteries ont continué lors de la visite du Premier Ministre grec Alexis Tsipras à Bruxelles, au siège de la Commision Européenne, pour rencontrer le Président de la Commission Européenne Jean-Claude Juncker le 4 Février 2015. A Bruxelles, l'image du geste de Juncker de prendre par la main Alexis Tsipras souriant mais embarrassé pour l'accompagner au bureau a fait le tour du monde (fig.5). Le journaliste commente que les blagues vont et viennent puisqu'il s'agit encore une fois d'un geste hors protocole politique. Il est vrai que cette image était négative pour le jeune Premier Ministre Tsipras, récemment élu, mais aussi pour le vieux politicien Juncker qui incarnait le savoir-vivre politique européen.

Ce geste pourrait être interprété comme un geste de manipulation du Premier Ministre grec pour le faire changer d'idée de ne pas payer la dette du pays et d'adopter "la politique réaliste," une politique de soumission aux marchés internationaux et à l'Union Européenne (à l'Allemagne particulièrement) comme l'avaient fait ses prédécesseurs. Le journaliste du média français L'Opinion, Gilles Sengès (2015), parle de "la diplomatie grecque à cols ouverts" soulignant qu' elle rappelle "celle des Bourgeois de Calais qui se livrèrent la nuque dégagée aux troupes d'Edouard III, en août 1347, dans le but de sauver la vie des habitants de la ville assiégée avant d'être épargnés grâce à l'intervention de Philippa de Hainaut, l'épouse du roi d'Angleterre." ${ }^{18}$ Cependant, cette image a fait le tour du monde dans les médias, ajoutant encore un moment embarrassant pour le gouvernement grec de l'époque.

Dans une autre rencontre politique, cette fois en Italie, le journaliste souligne le talent communicatif du Premier Ministre italien Matteo Renzi qui a offert à Alexis Tsipras une cravate (fig.6). Pour lui, à part sa courtoisie, le premier ministre Italien a eu comme but de montrer à nouveau que le Premier Ministre grec devait changer d'attitude et

\footnotetext{
${ }^{18}$ A voir, https: / / bit.ly / 3b4wpQw (dernière consultation le 16.12.2020).
} 
collaborer avec les autres pays membres de la zône euro. Selon le journaliste, puisque l'absence de cravate ${ }^{19}$ étant devenue un symbole du gouvernement, le forcer est ennuyeux. Il se demande si la prochaine étape sera un chapeau en cadeau.

Cette remarque n'est pas si innocente. Mettre à quelqu'un un chapeau connote un acte de supériorité dans la pratique grecque, peut-être dans le reste de l'Europe aussi. Il est aussi à noter les paroles du Premier Ministre italien pendant cette conférence: "nous voulons aider la Grèce, mais nous voulons aussi que le moment soit venu pour la Grèce de sortir de la crise, et lorsque ce-ci sera sortie de la crise, alors Alexis portera une cravate italienne." Ainsi, lorsque la Grèce sortira de la crise, le Premier Ministre grec pourra accompagner cet événement par un acte de haute esthétique, une cravate italienne qui connote le bon goût de l'industrie italienne de la mode. Il est à noter ici l'utilisation du prénom ${ }^{20}$ par le journaliste du Premier Ministre grec, une pratique qui n'est pas largement adoptée étant donné qu'elle montre un certain manque de respect pour la personne à qui on se réfère.

Quelque soit la relation entre les deux jeunes Premier Ministres, le protocole exige une image publique précise. L'utilisation du prénom du Premier Ministre grec et l'offre d'un cadeau plus personnelle ne sont pas passés inaperçues par les journalistes grecs et étrangers qui ont utilisé des commentaires vifs pour décrire cette ambiance légère.

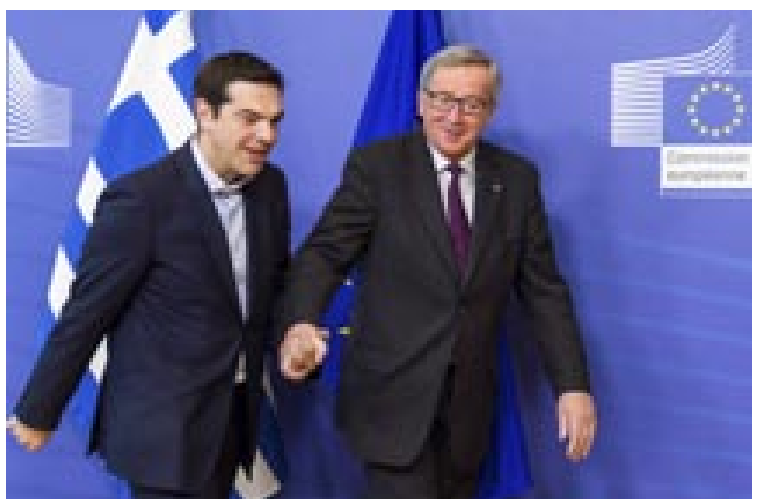

Figure 5. Visite du Premier Ministre grec Alexis Tsipras au siège de la Commission Européenne $(4.2 .2015)$

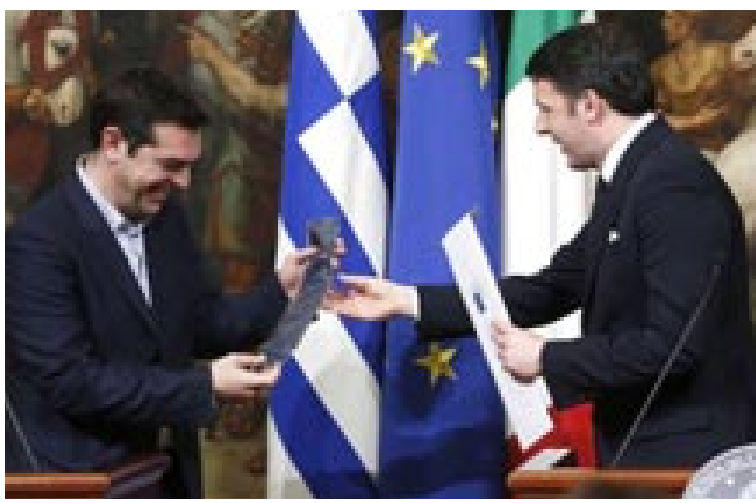

Figure 6. Le Premier ministre grec Alexis Tsipras reçoit le cadeau de Matheo Renzi (4.2.2015)

Les événements embarrassants des politiciens grecs avec les hauts dignitaires européens non seulement ne cessaient pas mais au contraire se multipliaient. Le 22 mai 2015, le Président de la Commission Européenne Jean Claude Juncker, a placé sa cravate sur son homologue grec, Alexis Tsipras proposant apparemment au Premier Ministre

\footnotetext{
${ }^{19}$ Devecchio (2019) remarque que "certes, il ne porte toujours pas de cravate, mais comme le fait remarquer une analyste politique, il a ajouté des 'pochettes à ses costumes', ce qui montre une adoption oblique du code vestimentaire soigné pour ses rencontres officielles."

${ }^{20}$ Il est à noter la position de Barthes (1972: 22) selon laquelle "le nom propre est lui aussi un signe [...] c'est un signe volumineux, un signe toujours gros d'une épaisseur touffue de sens [...]."
} 
grec, arrivé sans cravate, de lui prêter la sienne (fig. 7). Cette remarque du journaliste connote que le haut dignitaire européen, politique depuis 35 ans et Premier Ministre du Luxembourg pendant 19 ans, connaissait très bien le protocole politique et que peutêtre il y avait un sens caché dans son geste. Il est à noter que cette fois l'incident avait eu lieu lors de la photo officielle de tous les chefs des états et des gouvernements qui participaient au Sommet de l'Union Européenne.

Le langage du corps et surtout les incidents haptiques de la part des dirigeants de l'Union Européenne vers les politiciens grecs devenaient de plus en plus imprévisibles et choquants. Le 22 Juin 2015, Jean-Claude Juncker, le Président de la Commission européenne, tapotait gentiment la joue du Premier Ministre Alexis Tsipras, lors de la pause d'une conférence de presse conjointe (fig.8). En Grèce, mais aussi dans d'autres pays, il s'agit d'un geste entre un adulte et un enfant pour lui exprimer sa sympathie. Le fait que le Président de la Commission soit beaucoup plus âgé que le Premier Ministre grec pourrait aussi connoter l'immaturité politique de ce dernier. Sans doute il s'agit d'un geste public qui diminuait le statut politique et le sérieux du Premier Ministre grec, d'autant plus que cela se produisait devant les journalistes qui s'empressèrent d'immortaliser l'instantané.

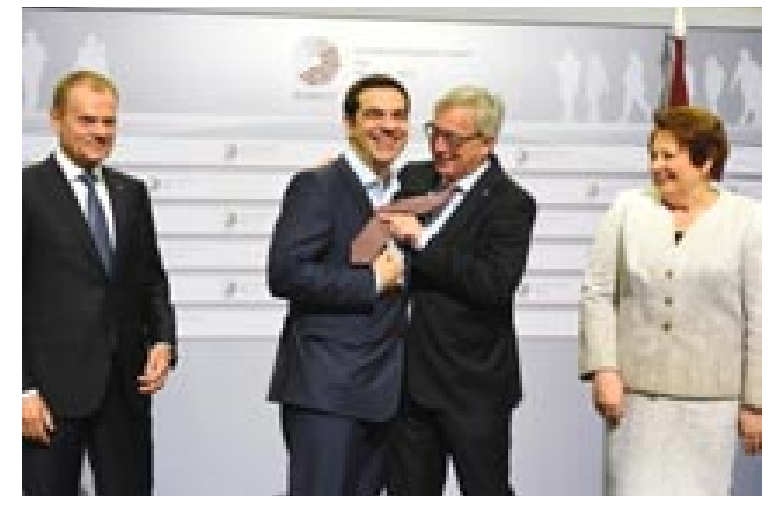

Figure 7. Le Premier Ministre grec Alexis Tsipras et le Président de la Commission JeanClaude Juncker (22.5.2015)

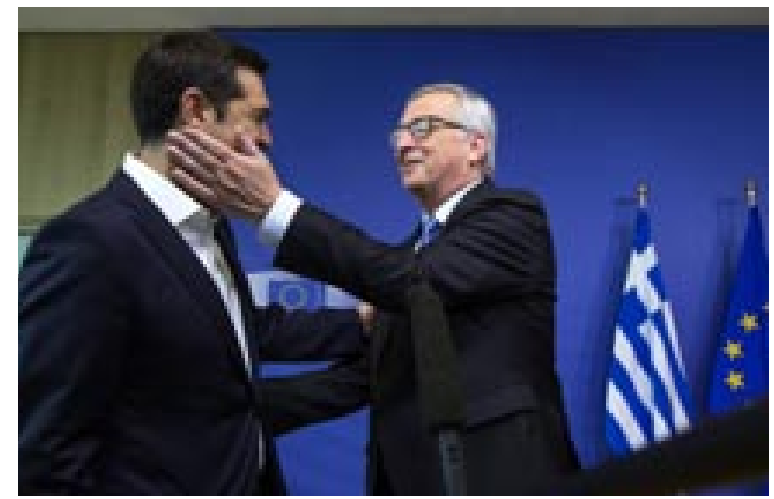

Figure 8. Alexis Tsipras et Jean-Claude Juncker $(22.6 .2015)$

\section{L'approche des événements par la presse grecque}

La presse grecque semblait confuse devant tous ces événements. Sans aucun doute, l'interprétation de ces événements était une tâche difficile. Lamizet (2006) mentionne que:

comprendre les représentations de l'événement ${ }^{21}$ dans les médias, c'est ainsi comprendre comment se forment notre culture, notre mémoire et notre enga-

\footnotetext{
${ }^{21}$ Selon Sini (2015 : 3), “la notion d"événement' ne renvoie qu'a une unité ambiguë, une substance glissante, délicate à circonscrire, dans la mesure où on ne sait pas quel objet il recouvre."
} 
gement politique dans l'espace de la sociabilité: la sémiotique de l'événement est nécessairement une sémiotique du politique, car elle nous amène à interroger de façon critique le sens des médiations constitutives de notre appartenance et de notre sociabilité. (Lamizet 2006: 18)

Dans un tel cadre, certains journalistes affrontent d'une manière légère la situation et, d'autres, ceux adoptent une position critique. Il ne faut pas oublier que:

La teneur d'un événement se saisit au travers de ses conséquences factuelles et des résonances qu'il crée auprès des acteurs sociaux. Il provoque des réactions souvent inattendues et imprédictibles auprès de l'opinion publique, à commencer par les journalistes ou les historiens eux-mêmes et leurs auditeurslecteurs, en passant par les décideurs politiques. (Sini 2015: 7)

Ainsi, selon le journaliste du journal en ligne iefimerida (22 mai 2015), certains voient que la disposition bienveillante de Juncker cache l'intention d'influencer le jeune nouveau de l'Eurogroupe. ${ }^{22}$ En même temps, pour le journaliste de l'édition électronique de la chaîne privée grecque Antenna (22 mai 2015), malgré la gravité de la situation, certains trouvent à plaisanter. L'un d'eux est le président de la Commission, qui continue ses... blagues. ${ }^{23} \mathrm{Il}$ est à noter que le titre même de l'article ne flatte pas le Premier Ministre grec.

L'intérêt pour ces événements dépasse les frontières grecques. Il ne faut pas oublier que "le geste est mémorable bien au-delà de son inscription; il traverse la recomposition narrative, il participe d'une scène actantielle mais laisse entrevoir toute l'épaisseur potentielle de l'acteur et tout le possible qui se situe au-delà des cadres systématisés des actes codés" (Basso Fossali 2017: 3). C'est pour cela que le journal anglais Gardian s'est adressé à un expert en langage du corps pour interpréter le geste de Juncker quand celui-ci tient la main de Tsipras. Selon le Gardian (2015), c'est Juncker qui prend l'initiative de tenir la main de Tsipras adoptant ainsi une position dominante de contrôle, un peu comme un parent avec un enfant. ${ }^{24}$

\section{Le reflet de l'information à d'autres systèmes sémiotiques}

L'aspect communicatif de l'art est relevé dans plusieurs études, non seulement sémiotiques. Une œuvre artistique ayant un but communicatif est sans doute la caricature de presse. Lotman (2004: 198) mentionne qu' “on pourrait distinguer deux tendances inverses dans le phénomène de l'art: celle qui consiste à répéter le déjà connu et celle qui

\footnotetext{
${ }^{22}$ Source: iefimerida.gr - https: / / bit.ly / 3pf6I4U (dernière consultation le 26.11.2020).

${ }^{23}$ https:/ / bit.ly/2Zeuzam (dernière consultation le 16.12.2020).

${ }^{24}$ A voir, https:/ / bit.ly / 3b8EEuS (dernière consultation le 16.12.2020).
} 
consiste à créer du nouveau." Les caricatures de presse que nous allons étudier appartiennent à la première classification de Lotman. Elles profitent des instantanés des rencontres politiques entre le gouvernement grec de l'époque et les dignitaires européens se transformant en multiplicateurs des interprétations que la mémoire collective grecque n'avait pas encore éclaircies. Sans doute, elles sont des réactions à l'information et Lotman mentionne que :

une nouvelle étape commence avec l'apparition du décalage temporel entre la réception de l'information et la réaction à celle-ci. Cette étape demande, avant tout, le développement et le perfectionnement de la mémoire. Un autre résultat très important est la transformation de la réaction de l'action immédiate en signe. La réaction à l'information devient une structure indépendante, susceptible de s'accumuler, avec un mécanisme qui se complexifie et se développe. (Lotman 2004: 183)

Une telle transformation en signe est le cas de la caricature de presse. La caricature a été utilisée par la presse de l'époque comme un moyen de reproduction des connotations des incidents des dignitaires européens avec le nouveau gouvernement grec. On pourrait, ainsi, prétendre qu'elle a été utilisée comme une sorte de dispositif de l'évocation de la mémoire politique qui était pour le peuple grec-avant tout-culturelle. Il est vrai que ce mécanisme humoristique a rendu la situation politique en Grèce plus complexe réservant à l'actualité des interprétations que le gouvernement grec de l'époque souhaitait sinon se débarrasser au moins annuler.

La caricature de presse est un moyen particulier de communication massive puisque la transmission efficace du message - qui est un message, d'habitude, implicite- est la composante des différents systèmes sémiotiques participant à la codification de l'humour. Exercice journalistique à part entière, la caricature de presse constitue de ce fait un instrument très efficace de commentaire d'analyse de l'actualité. Selon Charaudeau (2006: 11), la caricature de presse joue un rôle de catharsis sociale et de provocation sociale, elle n'est jamais anodine et son habillage humoristique ne peut exonérer son énonciateur.

Tous ces événements où les politiciens grecs refusent pour des raisons idéologiques de porter une cravate, a donné naissance à des caricatures de presse humoristiques comme les suivantes. La première caricature publiée dans L'Echo (31.1.2015) représente la chancelière allemande Angela Merkel se servant de la cravate comme instrument de strangulation du plus faible (fig. 9). La deuxième caricature publiée dans Placides Actualité (5.2.2015) fait allusion à une ceinture hypothétique offerte par la chancelière allemande à Tsipras et faisant allusion à l'expression "se serrer la ceinture" (fig.10). Il est à mentionner que cette interpretation a été reproduite dans la presse grecque. ${ }^{25}$

\footnotetext{
${ }^{25}$ A voir, https: / / bit.ly / 2N6m7HI (dernière consultation le 16.12.2020).
} 


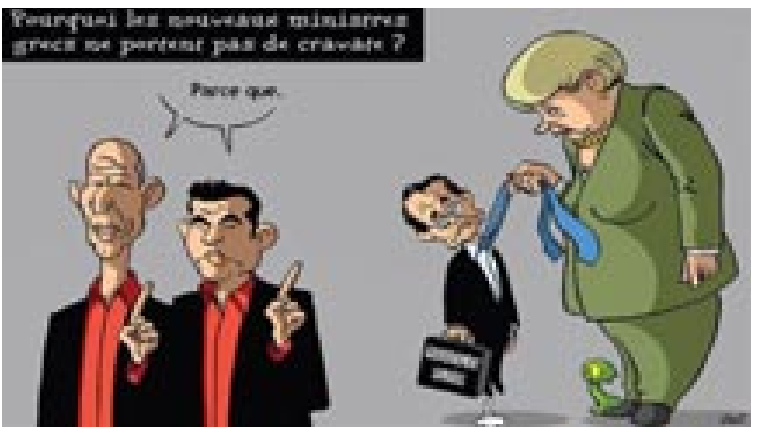

Figure 9. Dessin de Nicolas Vadot publié dans L'Echo (31.1.2015)

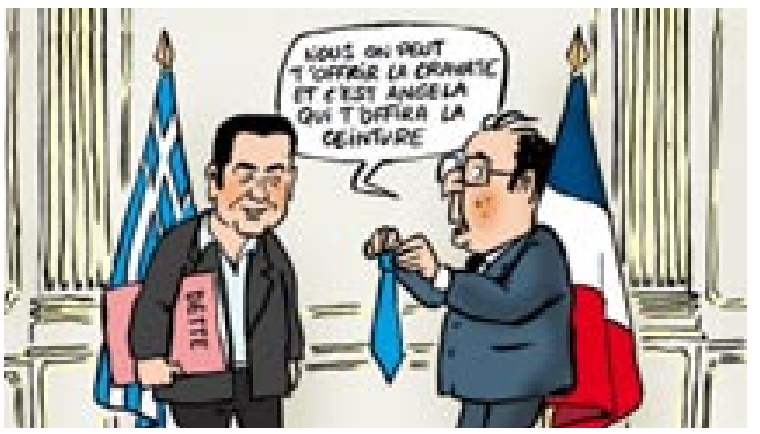

Figure 10. Dessin issu de Placides Actualité $(5.2 .2015)$

Cependant, les dessins humoristiques ne traitent pas seulement de l'absence de cravate parmi les membres du gouvernement grec de l'époque, notamment du Premier Ministre. Dans la caricature de presse suivante (fig.11) publiée dans The Guardian le 24 juin $2015^{26}$, des dignitaires européens se présentent inquiets lors des négociations sur la dette grecque parce que le Président de la Commission Européenne n'a pas tapoté cette fois la joue du Premier Ministre grec. Leur interprétation sémiotique est que Jean Claude Juncker est furieux contre Alexis Tsipras. Cette interprétation connote que l'évènement qui avait précédé a été jugé comme un acte de satisfaction politique envers le Premier Ministre grec et pas comme l'avait interprété une partie de la presse dévalorisant.

L'intimité dont les dignitaires européens font preuve envers le Premier Ministre grec se reflète aussi dans les dessins humoristiques. Dans la caricature publiée dans L'Opinion le 3 février 2015, ${ }^{27}$ le Président français François Hollande répondait à la question du Premier Ministre grec en utilisant son prénom, même si le Premier Ministre grec avait utilisé le vouvoiement (fig.12). Le fait que les deux hommes politiques se rencontrent dans un parc d'attractions rend la scène beaucoup plus amusante.

Cette intersémioticité qui se réalise entre le système sémiotique de la photo et celui de la caricature de presse montre que l'environnement politique de l'époque était très chargé -on pourrait dire à cause du rôle des médias dans la diffusion des interprétations politiques et culturelles dans la société. Pour Herzfeld (2016: 12), “[...] il semble que les caricatures sont non seulement acceptables mais remplacent les maigres miettes d'informations et de conjectures que les journalistes cherchent désespérément à glaner pour un public tout aussi désespéré". ${ }^{28}$ Pourtant, ces miettes d'information réservaient une polémique entre les deux parties des négociateurs pendant plus de six mois.

\footnotetext{
${ }^{26}$ A voir, https: / / bit.ly / 3d8y0HM (dernière consultation le 16.12.2020).

${ }^{27}$ A voir, https: / / bit.ly / 2OttARl (dernière consultation le 16.12.2020).

${ }^{28}[. .$.$] it seems, caricatures are not only acceptable but a substitute for the often meagre crumbs of information and$ guesswork that journalists are desperate to glean for an equally desperate public" (Herzfeld, 2016: 12).
} 


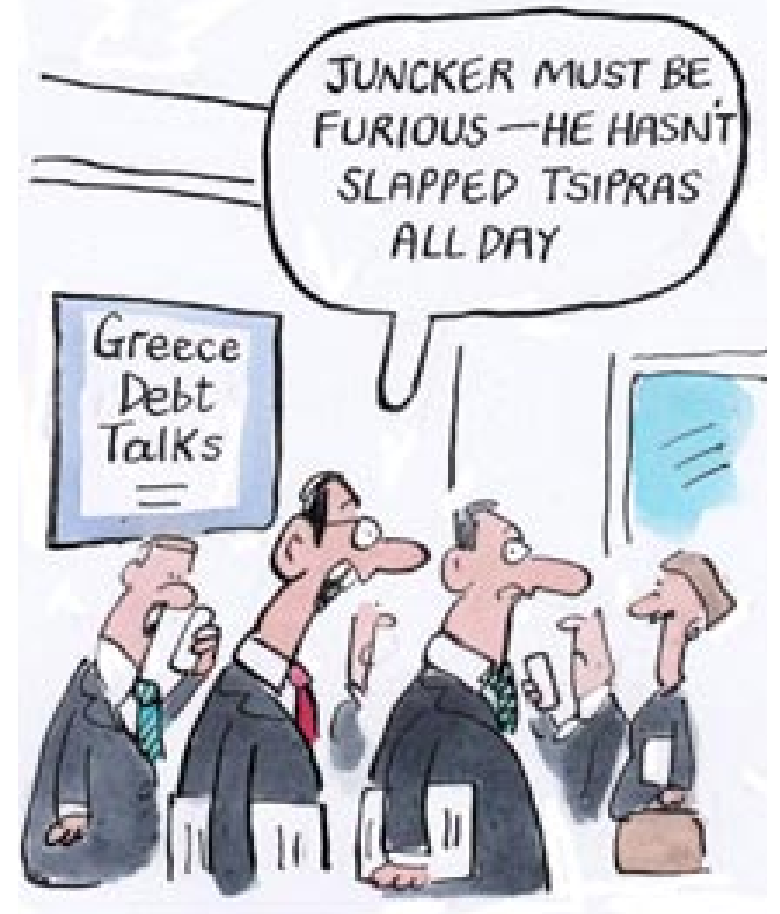

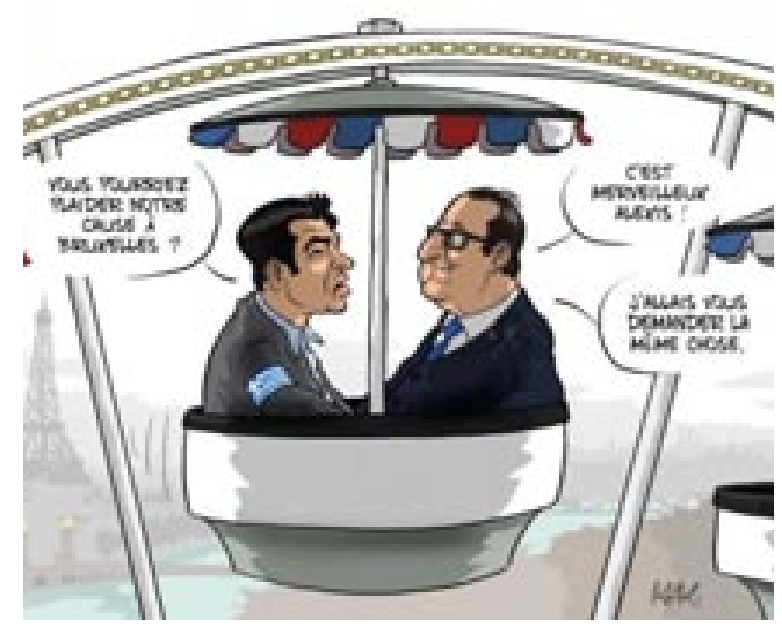

Figure 11 (left). Dessin de Kipper Williams publié dans The Guardian (24.6.2015)

Figure 12 (up). Dessin de Kak publié dans L'Opinion (3.2.2015)

\section{La sémiotique politique du réalisme?}

Nous avons vu que l'absence de cravate fonctionne au niveau sémiotique. Mais aussi son port après quelques années. Le 22 juin 2018, quand les créanciers de la Grèce ont déclaré la fin des programmes d'aide qui pesaient sur elle depuis 2010, une étape 'historique' avait eu lieu. Le Premier Ministre grec Alexis Tsipras avait souligné l'événement par le port inédit d'une cravate (fig. 13), tout en annonçant un tournant vers plus de justice sociale. Delafoi (2017) mentionne un fait historique lié à l'absence de cravate remarquant qu' "au début des années 1970, le président Mobutu, au Zaïre, a interdit le costume-cravate pour s'opposer à l'impérialisme occidental. Il est remplacé par l'abacost (contraction de «à bas le costume»), une veste de costume sans col". Il est à noter que la même attitude est aujourd'hui adoptée par les dignitaires de la République Islamique d'Iran.

Il est à souligner que le refus de porter une cravate dans la vie politique grecque avait été initié pendant la période de metapolitefsi (la période politique après la chute de la dictature grecque de 1967-1974 en Grèce connue aussi comme la Troisième République Hellénique). Cette apparence avait été adopté par le Premier Ministre socialiste Andreas Papadréou (fig.14). Papandréou qui ne portait pas ni chemise ni cravate, mais un pull à col roulé, style qui avait aussi suivi, au début, des députés de son parti politique PASOK (mouvement socialiste panhellénique). 


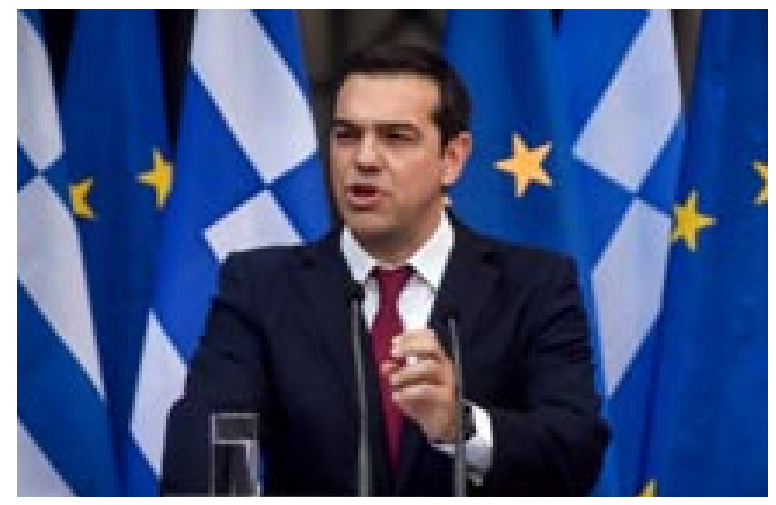

Figure 13. Le Premier Ministre grec Alexis Tsipras portant une cravate $(21.6 .2018)^{29}$

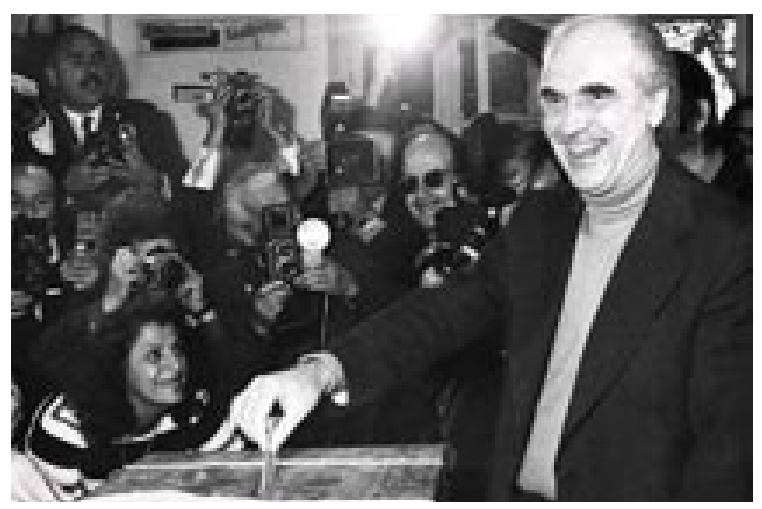

Figure 14. Le premier Ministre Grec Andreas Papandreou portant le pull à col roulé $(1974)^{30}$

Nous remarquons que les journalistes grecs reproduisent de temps en temps cette imitation (vestimentaire et kinésique) entre Tsipras et Papandréou reprochant au premier que ces similitudes sémiotiques ciblent le corps électoral du parti socialiste créé par le second. Un grand nombre de l'opinion publique considère que Tsipras valorise son statut par ces évocations sémiotiques étant donné que Papandréou était un universitaire et un politicien de renommée internationale.

Le port de cravate s'harmonise à une 'réalité', une norme, dans la vie politique qui demande aux politiciens de respecter le code vestimentaire. Pourtant, cette norme n'est pas arbitraire et va de pair avec un mode de vie et une façon de pensée politique précis. Quand les hommes politiques qui evitent au debut le port de cravate adoptent finalement ce style, ils donnent sémiotiquement l'impression qu'ils s'incorporent à une culture politique dominante, au savoir-vivre politique courant. 


\section{Constatations conclusives}

Il est vrai que la crise économique grecque (2010-2018) a inauguré un débat sur l'identité des pays du nord de l'Europe et de ceux dits méditerranéens. Le comportement des dignitaires européens était-il le résultat d'une autre identité qui ne partageait pas les mêmes caractéristiques culturelles avec les autres pays de l’Union européenne? Cet écart culturel se reflète-il dans le comportement des dignitaires européens vers les politiciens grecs?

Selon Lamizet (2012: 223), “[...] l'acteur politique fonde son identité sur la différenciation d'avec celle de l'autre et sur la confrontation avec elle. Dans ces conditions, l'imaginaire politique se trouve, lui aussi, fondé sur la notion d'adhésion et sur la confrontation avec les autres imaginaires." Toutes ces photos, reportages et caricatures de presse qui ont fait leur apparition dans la presse européenne connote que les politiciens grecs, dont il est question, n'appartiennent pas au groupe de la "famille européenne" parce qu'ils ne sont pas comme eux, ils se différencient au niveau des décisions politiques et, bien sûr, vestimentaires. Dans le meilleur des cas, ils sont des plaisantins, dans le pire des cas, ils sont des marginaux. C'est une des rare fois que le sérieux et la courtoisie qui caractérisaient le vie politique européenne des siècles précédents étaient absents.

Ce n'est pas une exagération de dire que les autorités de l'Union Européenne ont adopté un comportement qui était attribué par Barthes à la petite bourgeoisie. Selon Barthes (1957: 81), "[...] toute la mythologie petite-bourgeoisie implique le refus de l'altérité, la négation du différent, le bonheur de l'identité et l'exaltation du semblable". Les dirigeants de l'UE avec leur comportement ont voulu souligner le différent des idées politiques du gouvernement grec en question et de distinguer sémiotiquement la Grèce de l'époque des autres pays membres qui partageaient le semblable, les mêmes idées économiques et politiques.

A l'époque, en 2015, les autorités européennes voulaient souligner sémiotiquement le manque de maturité politique du nouveau gouvernement grec à qui les hauts dignitaires européens reprochaient son populisme et son manque de réalisme politique. Ainsi, au niveau sémiotique, on constate une double raillerie, l'une, vers le peuple grec qui traversait une crise économique grave, et l'autre, vers ses élus politiques, difficilement acceptés par les autres pays membres de la zône euro à cause de la politique qu'ils souhaitaient suivre.

Ce serait une omission de ne pas mentionner que cette gestualité a caractérisé seulement le gouvernement de gauche radicale de cette période. Quand le même parti politique a changé son orientation politique en ce qui concerne la dette grecque et qu'il prit la décision de collaborer harmonieusement avec les autorités de l'Union Européenne et les créditeurs du pays, tous ces événements ont disparu ce qui permet de 
supposer qu'il s'agissait de signes motivés, de signes intentionnels. De cette façon, il semble que si un nouveau gouvernement européen prenait des décisions politiques différentes de celles des autres pays membres, on vivrait à nouveau des situations embarrassantes au niveau sémiotique comme celles de 2015. Cette supposition nous invite à réfléchir sur le futur du rêve commun de l’Union Européenne, de réfléchir sur les valeurs et l'identité communes.

\section{Références bibliographiques}

Ahonen, Pertii 1991. Politics and generation of meanings: The contribution of the semiotics of Greimas. In: Jeff Bernard et al. (eds.) Theoretische und praktische Relevanz der Semiotik. Vienna: ISSS, 243-279.

Alonso, Juan 2014. Semiotique et politique: narrativite et transformation. Recherches en communication 41: 61-74.

Basso Fossali, Pierluigi 2017. Le geste et sa niche: gestion du sens 'hors technique'. Texto ! Textes et Cultures 22 (2): 1-27.

Barthes, Roland 1957. Mythologies. Paris: Editions du Seuil.

Barthes, Roland 1961. Le message photographique. Communications 1: 127-138.

Barthes, Roland 1967. Système de la Mode. Paris: Seuil.

Barthes, Roland 1972. Le degré zéro de l'écriture. Suivi de Nouveaux essais critiques. Paris: Editions du Seuil.

Barthes, Roland 1980. Roland Barthes par Roland Barthes. Paris: Seuil.

Barthes, Roland \& Olivier Burgelin 2001. Le bleu est à la mode cette année et autres articles. Paris: Editions de l'Institut Français de la Mode.

Bronwen, Martin \& Felizitas Ringham 2006. Key Terms in Semiotics. London \& New York: Continuum.

Bordron, Jean-François 2013. Image et vérité: Essais sur les dimensions iconiques de la connaissance. Liége: Presses universitaires de Liège. Disponible sur Internet: https: / / bit.ly / 3u0yXaT [généré le 16 décembre 2020].

Boutin, Christophe, Olivier Dard \& Frederic Rouvillois 2019. Dictionnaire des populismes. Paris: Editions du Cerf.

Calbris, Geneviève 2001. Principes méthodologiques pour une analyse du geste accompagnant la parole. Mots. Les langages du politique 67: 129-148.

Charaudeau, Patrick 2006. Discours journalistique et positionnement énonciatifs. Frontières et dérives. Semen 22: 1-13.

Daley, Suzanne 2015. A Grass-Roots Pitch in Greece Could Pay Off. The New York Times, January 17, https: / / nyti.ms/37cUbc8

Danesi, Marcel 2004. Messages, Signs, and Meanings: A Basic Textbook in Semiotics and Communication Theory. Toronto: Canadian Scholars' Press Inc. 
Delafoi, Florian.2017. Cravate et politique, la révolution des apparences. Le Temps, 20 Juillet, https: / / bit.ly/37ghLo7

Devecchio, Alexandre 2019. Recomposition. Le nouveau monde populiste. Paris: Editions du Cerf.

Drechsler, Wolfgang 2009. Political Semiotics. Semiotica 173 (1): 73-9.

Fabbri, Paolo 1968. Considérations sur la proxémique. Langages 10: 65-75.

Friedman, Vanessa 2015. The Wardrobe Politics of Greece's New Prime Minister. The New York Times, December 5, 2020, https: / / nyti.ms / 3q1QJ6z

Heslin, Richard 1974. Steps Toward a Taxonomy of Touching. In: Richard Heslin (ed.) Getting Close: Research and Theory on Spatial Distance, Touching, and Eye Contact. Paper presented at the Western Psychological Association Conference, Chicago, Illinois, 1974.

Herzfeld, Michael 2016. The hypocrisy of European moralism. Greece and the politics of cultural aggression - part I. Anthropology Today 32 (1): 10-13.

Greimas, Algirdas-Julien \& Joseph Courtés 1993. Sémiotique. Dictionnaire raisonné de la théorie du langage. Paris: Hachette.

Kourdis, Evangelos. 2016. The Velopoulos-Liakopoulos Phenomenon. A semiotic approach to the explosion of Greek conspiracy theories and urban legends in the economic crisis. Lexia-Revista di Semiotica 23-24: 225-244.

Lamizet, Bernard 2002. Politique et identité. Presse Universitaire de Lyon.

Lamizet, Bernard 2006. Sémiotique de l'évènement. Paris: Lavoisier.

Lamizet, Bernard 2006b. La sémiotique instante: introduction à la sémiotique politique . Semiotica 159 (1): 1-54.

Lamizet, Bernard 2012. L'imaginaire politique. Paris Lavoisier.

Lamizet, Bernard \& Ahmed Silem (eds) 1997. Dictionnaire encyclopédique des sciences de l'information et de la communication. Paris: Ellipses.

Lotman, Jouri 2004. L'explosion et la culture. Limoges: Pulim.

Lotman, Juri 2013. The Unpredictable Workings of Culture. Tallinn: Tallinn University Press.

Mayi, Joseph 2016. Images du pouvoir et pouvoirs de l'image. La peopolisation, un dispositif social et technique au service de la construction des normes de Genre en politique. Le cas de la scène politique française de 2002 à 2012. Nice: Université Nice Sophia Antipolis.

Owyong, Yuet See Monica 2009. Clothing semiotics and the social construction of power relations. Social Semiotics 19 (2): 191-211.

Sini, Lorella 2015. Evènements, discours, médias: réflexions à partir de quelques travaux récents. Argumentation et Analyse du Discours 14: 1-17. 
Sullivan, Antony 2019. Dressing the Opposition: Sartorial Resistance on Europe's Political Left. In: Djurdja Bartlett (ed.) Fashion and Politics. New Haven and London: Yale University Press, 167-177.

Turpin, Béatrice 2016. Pour une sémiotique du politique: schèmes mythiques du national-populisme. Semiotica 159 (1): 285-304.

\section{AUTEUR \\ Evangelos Kourdis Professor of Translation Semiotics, School of French Language and Literature, and Director of the Laboratory of Semiotics (AUTH SemioLab), Fac- ulty of Philosophy, Aristotle University of Thessaloniki, Greece.}




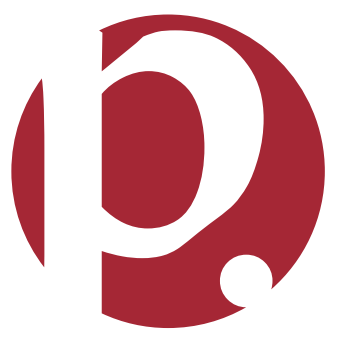

